\title{
Semi-discrete Schemes for Hamilton-Jacobi Equations on Unstructured Grids
}

\author{
Doron Levy ${ }^{1}$ and Suhas Nayak ${ }^{2}$ \\ 1 Department of Mathematics, Stanford University, Stanford, CA 94305-2125, USA \\ dlevy@math.stanford.edu \\ 2 Department of Mathematics, Stanford University, Stanford, CA 94305-2125, USA \\ snayak@stanford.edu
}

Summary. We present a new semi-discrete central scheme for approximating solutions of Hamilton-Jacobi equations on unstructured meshes. This scheme extends the numerical Hamiltonians of Kurganov et al. to unstructured grids. Similarly to the previous works on structured grids, a semi-discrete formulation of central schemes is made possible due to estimates of the local speeds of propagation. The consistency of the method is obtained following Abgrall's calculations for the consistency of an upwind Lax-Friedrichs scheme on unstructured grids. We conclude with comments on high-order reconstructions.

\section{Introduction}

We present a new central-upwind scheme for approximating solutions of Hamilton-Jacobi (HJ) equations on unstructured grids. These equations are of the form

$$
\phi_{t}+H(\nabla \phi)=0, \quad \mathbf{x}=\left(x_{1}, \ldots x_{d}\right) \in \mathbb{R}^{d},
$$

where $\phi=\phi(\mathbf{x}, t)$, and with a Hamiltonian, $H$, that depends on $\nabla \phi$ and possibly on $x$ and $t$. Since solutions of (1) may develop discontinuous derivatives even when the initial data is smooth, it is generally required to interpret the solution of (1) in a suitable weak sense. The corresponding theory, in the form of "viscosity solutions" has been significantly developed over the past two decades and we refer to $[5,6,14,15]$.

The increased understanding of the nature of solutions to HJ equations have turned the area of numerical methods for the HJ equations into an active research area. Converging first-order methods were introduced by Souganidis [19]. The order of accuracy of the methods was increased through an essentially non-oscillatory (ENO) reconstruction in the upwind schemes of Osher, Sethian and Shu [17, 18]. Weighted essentially non-oscillatory (WENO) reconstructions, which were first introduced for hyperbolic conservation laws $[8,16]$, were then used by Jiang and Peng [7] to even further increase the accuracy of the numerical approximations using a compact reconstruction. Extensions of the first-order and ENO upwind methods to unstructured grids were done by Abgrall [1]. The numerical fluxes of Abgrall were combined with WENO reconstructions on triangular meshes by $\mathrm{Hu}$ and $\mathrm{Shu}$ in [20]. Another finite-volume scheme on unstructured grids was proposed by Kossioris et al. in [9].

While upwind schemes require solving Riemann problems (or at least approximating their solutions) on the interfaces between two cells, Godunov-type central schemes utilize evolution points that are located away from the discontinuous interfaces in order to avoid Riemann solvers altogether. Fully-discrete central schemes for HJ equations were first introduced by Lin and Tadmor in $[12,13]$, and further improved by Bryson and 
Levy in [2]. Fully-discrete schemes of order greater than two were derived in [3] using central-WENO reconstructions. Semi-discrete formulations of central schemes enjoy reduced numerical dissipation while keeping track over the local speeds of propagation of information that is propagating from the discontinuous interfaces. Second-order semidiscrete central schemes for HJ equations were derived by Kurganov and Tadmor in [11]. A more accurate estimate of the local speed of propagation was then utilized to reduce the numerical dissipation in [10]. The numerical flux of Kurganov, Noelle and Petrova, was combined with the WENO reconstructions of Jiang and Peng to obtain fifth-order, semi-discrete central schemes for multi-dimensional HJ equations in [4].

In this paper we extend our previous works on semi-discrete central schemes for multi-dimensional HJ equations to unstructured grids. Our derivation results with a new numerical flux that takes into account information regarding the local speeds of propagation of information from the discontinuous interfaces. Similarly to any other central scheme, the evolution points are taken away from the interfaces in order to avoid Riemann solvers. This numerical Hamiltonian should be viewed as a central version of the numerical Hamiltonian of Abgrall [1]. It can also be viewed as a generalization of the numerical Hamiltonians of Kurganov et al. $[10,11]$ that were derived for Cartesian grids. When we assume that the "unstructured" grid is Cartesian (which still is an admissible grid in our formulation) the scheme we obtain is different of the schemes obtained in $[11,10]$. This difference results from a different averaging procedure we use with the values obtained at the different evolution points, and is reflected by a different coefficient in front of the dissipative term.

\section{A Semi-discrete scheme for HJ equations}

We consider two-dimensional Hamilton-Jacobi equations of the form

$$
\phi_{t}+H(\nabla \phi)=0, \quad \mathbf{x}=\left(x_{1}, x_{2}\right) \in \Omega \subset \mathbb{R}^{2},
$$

augmented with initial values $\phi(x, t=0)=\phi_{0}(x)$. We assume that $\mathcal{T}$ is a given triangulation of $\Omega$. The grid points are denoted by $x_{\alpha}$. For every grid point there are $m_{\alpha}$ angular sectors $T_{l}^{\alpha}$ that are ordered counterclockwise. For simplicity we will drop the $\alpha$ index from the triangles, and use the notation $T_{l}=T_{l}^{\alpha}$ whenever possible. Each node of our triangulation may be visualized as in Figure 1.

The semi-discrete scheme will be constructed as a limit of a fully-discrete scheme as the time-step tends to zero. We therefore assume a time step, $\Delta t$, and use the standard notation $t^{n}=n \Delta t$. Let $\varphi_{\alpha}^{n}$ denote the approximate value of $\phi\left(x_{\alpha}, t^{n}\right)$. We assume that for each time $t^{n}, \varphi_{\alpha}^{n}$ is given at each node $x_{\alpha}$ of the unstructured grid. We also assume that the values $\varphi_{\alpha}^{n}$ can be used to reconstruct a continuous piecewise-polynomial interpolant, i.e., a polynomial in each sector. We will comment below on a method for obtaining such a reconstruction. In either case, the numerical flux we develop is independent of the reconstruction step. We denote this reconstruction by $\tilde{\varphi}_{\alpha}$ and denote the approximation of $\nabla \varphi$ in each sector $\nabla \tilde{\varphi}_{\alpha, l}$.

The second ingredient we need is an estimation of the maximal speeds of propagation at the interfaces of each angular sector (in a direction that is perpendicular to the interface). For any given angular sector, $T_{l}$, the counterclockwise speed of propagation is denoted by $a_{l}^{+}$and the speed of propagation on the other interface is $a_{l}^{-}$. These speeds can be estimated by: 


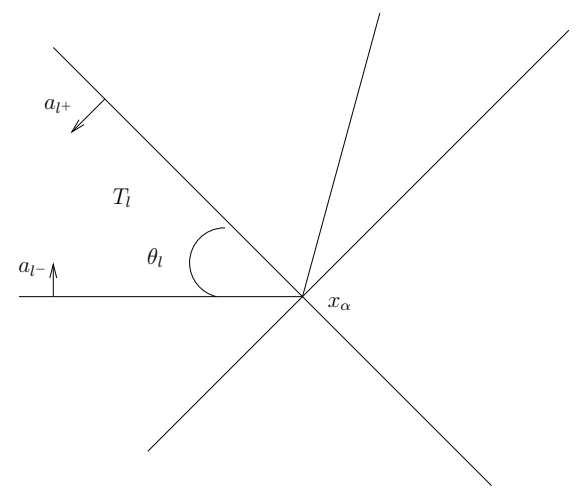

Fig. 1. Grid point $x_{\alpha}$ and its angular sectors. Each angular sector $l$ has an associated angle $\theta_{l}$

$$
\begin{aligned}
& a_{l}^{+}=\max \left\{\max _{T_{l}}\left\{\nabla H\left(\nabla \varphi_{\alpha, l}\right) \cdot \mathbf{n}_{l-1, l}\right\}, \max _{T_{l-1}}\left\{\nabla H\left(\nabla \varphi_{\alpha, l-1}\right) \cdot \mathbf{n}_{l-1, l}\right\}\right\}, \\
& a_{l}^{-}=\max \left\{\max _{T_{l}}\left\{\nabla H\left(\nabla \varphi_{\alpha, l}\right) \cdot \mathbf{n}_{l+1, l}\right\}, \max _{T_{l+1}}\left\{\nabla H\left(\nabla \varphi_{\alpha, l+1}\right) \cdot \mathbf{n}_{l+1, l}\right\}\right\},
\end{aligned}
$$

where $\mathbf{n}_{j, l}$ is the normal vector on the interface between sectors $T_{j}$ and $T_{l}$ pointing into $T_{l}$.

We can now determine in every sector $T_{l}$ around $x_{\alpha}$ an evolution point $x_{\alpha}^{l}$ that is located away from the interfaces (see Figure 2). This will be done using the distances that are defined through the local speeds of propagation $a_{l}^{ \pm}$.

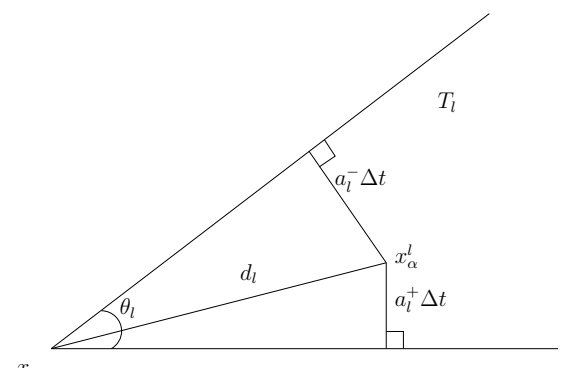

Fig. 2. Evolution point, $x_{\alpha}^{l}$, derived from the maximal local speeds of propagation into $T_{l}, a_{l}^{+}$ and $a_{l}^{-}$.

The distance of the evolution point $x_{\alpha}^{l}$ from $x_{\alpha}$ is denoted by $d_{l}$. Clearly, $d_{l}$ depends on the local speeds of propagation $a_{l}^{ \pm}$and on the angle $\theta_{l}$ and is given by

$$
d_{l}^{2}=\frac{\left(a_{l}^{-} \Delta t\right)^{2}+2 a_{l}^{-} a_{l}^{+} \Delta t^{2} \cos \theta_{l}+\left(a_{l}^{+} \Delta t\right)^{2}}{\sin ^{2} \theta_{l}} .
$$

We then define $\hat{d}_{l}$ to be $d_{l} / \Delta t$, a quantity that does not explicitly depend on $\Delta t$.

The interpolant $\tilde{\varphi}\left(\mathbf{x}, t^{n}\right)$ is evolved to the next time step $t^{n+1}$ at the points $x_{\alpha}^{l}$, which are located away from the propagating discontinuities (assuming that the time step $\Delta t$ is sufficiently small). From (1), this is given to first order in time by the Taylor expansion

$$
\varphi\left(x_{\alpha}^{l}, t^{n+1}\right)=\tilde{\varphi}\left(x_{\alpha}^{l}, t^{n}\right)-\Delta t H\left(\nabla \tilde{\varphi}\left(x_{\alpha}^{l}, t^{n}\right)\right)+O\left(\Delta t^{2}\right),
$$


where the approximation of the gradient $\nabla \varphi$ at $x_{\alpha}^{l}, \nabla \tilde{\varphi}\left(x_{\alpha}^{l}, t^{n}\right)$, is obtained from the reconstruction.

The next step is to combine the values of $\varphi$ at the different evolution points around $x_{\alpha}, x_{\alpha}^{l}$, into one value $\varphi_{\alpha}^{n+1}$. This is done by writing a convex combination with weights $s_{l} \geq 0$ that are yet to be determined:

$$
\varphi_{\alpha}^{n+1}=\frac{\sum_{l=1}^{m_{\alpha}} s_{l} \varphi\left(x_{\alpha}^{l}, t^{n+1}\right)}{\sum_{l=1}^{m_{\alpha}} s_{l}} .
$$

Using (4), we may express (5) as

$$
\varphi_{\alpha}^{n+1}=\frac{\sum_{l=1}^{m_{\alpha}} s_{l}\left[\tilde{\varphi}\left(x_{\alpha}^{l}, t^{n}\right)-\Delta t H\left(\nabla \tilde{\varphi}\left(x_{\alpha}^{l}, t^{n}\right)\right]\right.}{\sum_{l=1}^{m_{\alpha}} s_{l}} .
$$

If we now define $\rho_{l}$ to be the unit vector in the direction of $x_{\alpha}^{l}$ from $x_{\alpha}$, we can use a Taylor expansion in space

$$
\tilde{\varphi}\left(x_{\alpha}^{l}, t^{n}\right)=\tilde{\varphi}\left(x_{\alpha}, t^{n}\right)+d_{l} \rho_{l} \cdot \nabla \tilde{\varphi}\left(x_{\alpha}^{l}, t^{n}\right)+O\left(\Delta t^{2}\right) .
$$

Here by $\nabla \tilde{\varphi}\left(x_{\alpha}^{l}, t^{n}\right)$ we refer to the value of the gradient at $x_{\alpha}$ that is associated with the reconstruction in sector $T_{l}$ at $x_{\alpha}^{l}$. We may therefore rewrite (6) as the fully discrete scheme

$$
\varphi_{\alpha}^{n+1}=\tilde{\varphi}_{\alpha}^{n}+\frac{\Delta t}{\sum_{l=1}^{m_{\alpha}} s_{l}} \sum_{l=1}^{m_{\alpha}} s_{l}\left[\hat{d}_{l} \rho_{l} \cdot \nabla \tilde{\varphi}\left(x_{\alpha}^{l}, t^{n}\right)-H\left(\nabla \tilde{\varphi}\left(x_{\alpha}^{l}, t^{n}\right)\right)\right] .
$$

In the limit $\Delta t \rightarrow 0,(7)$ becomes a semi-discrete scheme:

$$
\frac{d}{d t} \varphi_{\alpha}(t)=\frac{1}{\sum_{l=1}^{m_{\alpha}} s_{l}} \sum_{l=1}^{m_{\alpha}} s_{l}\left[\hat{d}_{l} \rho_{l} \cdot \nabla \tilde{\varphi}_{\alpha}^{l}(t)-H\left(\nabla \tilde{\varphi}_{\alpha}^{l}(t)\right)\right],
$$

where for each $l, \nabla \tilde{\varphi}_{\alpha}^{l}(t)$ denotes $\lim _{\Delta t \rightarrow 0} \nabla \tilde{\varphi}\left(x_{\alpha}^{l}, t^{n}\right)$. All that remains is to determine the coefficients $s_{l}$ in (8). These coefficients will be obtained through a consistency condition. The consistency of the scheme implies that if the value of the gradient is identical in every sector that surrounds $x_{\alpha}$ then the numerical Hamiltonian should reduce to the differential Hamiltonian. Hence we are seeking for coefficients $s_{l}$ such that

$$
\sum_{l=1}^{m_{\alpha}} s_{l} \hat{d}_{l} \rho_{l}=0
$$

Such coefficients indeed exist, and we can use, e.g., the results of Abgrall in [1] to find them. The observation that was made there was that if $\mu_{l+1 / 2}$ denoted a unit vector in a direction that is aligned with the interface between the sectors $T_{l}$ and $T_{l+1}$, and if $\theta_{l}<\pi$ (which is the case with a triangulation, e.g.), then

$$
\sum_{l=1}^{m_{\alpha}} \gamma_{l+\frac{1}{2}} \mu_{l+\frac{1}{2}}=0
$$

provided that 


$$
\gamma_{l+\frac{1}{2}}=\epsilon\left[\tan \left(\frac{\theta_{l}}{2}\right)+\tan \left(\frac{\theta_{l+1}}{2}\right)\right]
$$

for any $\epsilon>0$. In order to incorporate (10)-(11) into our framework, we split each angle $\theta_{l}$ into two parts $\theta_{l}^{ \pm}$that are defined as

$$
\theta_{l}^{ \pm}=\arcsin \frac{a_{l}^{ \pm}}{\hat{d}_{l}}
$$

(see figure 3).

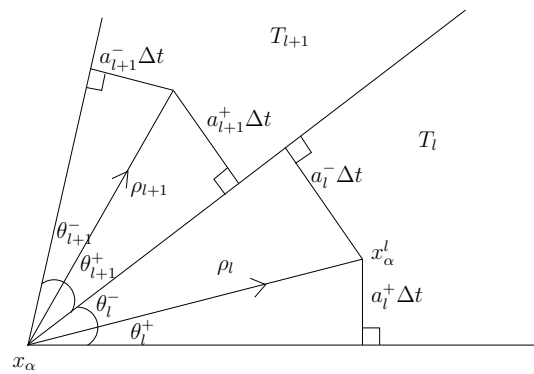

Fig. 3. The angles around $x_{\alpha}$

The consistency condition (9) is then satisfied if the weights $s_{l}$ are defined as

$$
s_{l}=\frac{\epsilon}{\hat{d}_{l}}\left[\tan \left(\frac{\theta_{l}^{+}+\theta_{l-1}^{-}}{2}\right)+\tan \left(\frac{\theta_{l}^{-}+\theta_{l+1}^{+}}{2}\right)\right],
$$

where $\theta_{l}^{ \pm}$are given by (12). In our case, the coefficient $\epsilon$ will anyhow cancel out in the semi-discrete formulation (8), so it can be omitted from (13).

To summarize, if we define

$$
\beta_{l}=\left[\tan \left(\frac{\theta_{l}^{+}+\theta_{l-1}^{-}}{2}\right)+\tan \left(\frac{\theta_{l}^{-}+\theta_{l+1}^{+}}{2}\right)\right],
$$

then the semi-discrete scheme is given by

$$
\frac{d}{d t} \varphi_{\alpha}(t)=\frac{1}{\sum_{l=1}^{m_{\alpha}} \frac{\beta_{l}}{\hat{d}_{l}}} \sum_{l=1}^{m_{\alpha}} \beta_{l}\left[\rho_{l} \cdot \nabla \tilde{\varphi}_{\alpha}^{l}(t)-\frac{H\left(\nabla \tilde{\varphi}_{\alpha}^{l}(t)\right)}{\hat{d}_{l}}\right] .
$$

\section{Remarks.}

1. A simple version of the scheme can be obtained by assuming that all the speeds of propagation are identical. In this case, the local speeds are replaced by their maximum, i.e., $a=\max _{l}\left\{a_{l}^{+}, a_{l}^{-}\right\}$. In this case

$$
\hat{d}_{l}=\frac{a}{\sin \left(\theta_{l} / 2\right)}
$$

and the semi-discrete scheme (14) can be written in the simpler form 


$$
\frac{d}{d t} \varphi_{\alpha}(t)=\frac{a}{\sum_{l=1}^{m_{\alpha}} \beta_{l} \sin \frac{\theta_{l}}{2}} \sum_{l=1}^{m_{\alpha}} \beta_{l}\left[\rho_{l} \cdot \nabla \tilde{\varphi}_{\alpha}^{l}(t)-\frac{\sin \frac{\theta_{l}}{2}}{a} H\left(\nabla \tilde{\varphi}_{\alpha}^{l}(t)\right)\right] .
$$

If, in addition to the velocities being identical, the angles are also assumed to be identical, i.e. $\theta=\theta_{l}, \forall l$, then (15) can be further simplified into

$$
\frac{d}{d t} \varphi_{\alpha}(t)=\frac{1}{m_{\alpha}} \sum_{l=1}^{m_{\alpha}}\left[\frac{a}{\sin \frac{\theta}{2}} \rho_{l} \cdot \nabla \tilde{\varphi}_{\alpha}^{l}(t)-H\left(\nabla \tilde{\varphi}_{\alpha}^{l}(t)\right)\right] .
$$

2. In [1] Abgrall derived a Lax-Friedrichs-type scheme on triangular meshes. In our notation, his scheme is of the form

$$
\frac{d}{d t} \varphi_{\alpha}(t)=\frac{a}{\pi} \sum_{l=1}^{m_{\alpha}} \beta_{l+\frac{1}{2}} \rho_{l+\frac{1}{2}} \cdot\left(\frac{\nabla \tilde{\varphi}_{\alpha}^{l}(t)+\nabla \tilde{\varphi}_{\alpha}^{l+1}(t)}{2}\right)-H\left(\frac{\sum_{l=1}^{m_{\alpha}} \theta_{l} \nabla \tilde{\varphi}_{\alpha}^{l}}{2 \pi}\right) .
$$

Here $\rho_{l+1 / 2}$ is the unit vector in the direction of the interface between the sectors $T_{l}$ and $T_{l+1}$, and $\beta_{l+1 / 2}=\tan \left(\theta_{l} / 2\right)+\tan \left(\theta_{l+1} / 2\right)$. The derivation of (17) involved evolution points that were located on the interfaces between the sectors. This resulted with the form of the dissipative term in (17) that contains averages of gradients in adjacent sectors. Also, the scheme (17) involves a Hamiltonian that is evaluated at the average of the derivatives that are computed in different sectors (with weights that are proportional to the angles). This term was postulated to be in this form, and could have taken different forms. In our case (14), this term is replaced by an average over the Hamiltonian that is evaluated in different sectors. In our case, the form of this term is dictated by the derivation of the scheme.

3. We would like to emphasize that the scheme (14) does not require Riemann solvers. This is due to the scheme's derivation, which places the evolution points away from the boundaries of the angular sectors around every point $x_{\alpha}$.

4. If the grid is a Cartesian grid with equal spacing in the $x$ - and $y$-directions, the number of angular sectors at each point is $m_{\alpha}=4$, and $\sin (\theta / 2)=\sin (\pi / 4)=$ $\sqrt{2} / 2$. In the simple case where all velocities are taken to be identical in both direction, the scheme (16) becomes

$$
\begin{aligned}
& \frac{d}{d t} \varphi_{\alpha}(t)=\frac{a}{2}\left(\varphi_{x}^{+}-\varphi_{x}^{-}+\varphi_{y}^{+}-\varphi_{y}^{-}\right) \\
& \quad-\frac{1}{4}\left[H\left(\varphi_{x}^{+}, \varphi_{y}^{+}\right)+H\left(\varphi_{x}^{-}, \varphi_{y}^{+}\right)+H\left(\varphi_{x}^{+}, \varphi_{y}^{-}\right)+H\left(\varphi_{x}^{-}, \varphi_{y}^{-}\right)\right],
\end{aligned}
$$

with the obvious notations, e.g. $H\left(\varphi_{x}^{+}, \varphi_{y}^{+}\right)$is the Hamiltonian evaluated at the gradient at $x_{\alpha}$ that is taken from the first quadrant. The scheme (18) is identical to the consistent and monotone semi-discrete scheme that was derived for Cartesian grids in $[4,11]$.

5. The order of accuracy of the scheme (14) is determined by the order of accuracy of the reconstruction and the order of the ODE solver. Other than that, the formulation (14) is independent of the order of accuracy of the scheme. The scheme is a Godunov-type scheme with a global reconstruction that is evolved in time in evolution points that are located away from the interfaces. In practice, the final semi-discrete scheme (14) requires only the values of the gradient at the grid points $x_{\alpha}$ that are computed in the different angular sectors around $x_{\alpha}$. Hence, all that 
one needs from the reconstruction is the values of these gradients. It is therefore possible to use the same reconstructions that were developed for upwind schemes for HJ equations on triangular meshes. For examples, a high-order (third- or fourth-order) weighted essentially non-oscillatory (WENO) reconstruction for HJ equations on triangular grids was derived by Zhang and Shu [20]. It can be incorporated as it is into the present framework.

\section{Conclusion}

We have derived a new semi-discrete central scheme for HJ equations on unstructured grids. This scheme is a generalization of the semi-discrete central schemes on Cartesian grids $[4,10,11]$. It is a Godunov-type scheme where a global reconstruction is evolved in time and then projected back to the grid points. Since the evolution is performed away from the interfaces of the angular sectors, there is no need to use exact or approximate Riemann solvers. The formal accuracy of the scheme depends on the accuracy of the reconstruction and the order of the ODE solver being used.

\section{References}

1. Abgrall, R. (1996): Numerical discretization of the first-order Hamilton-Jacobi equation on triangular meshes. Commun. Pure Appl. Math., 49, 1339-1373.

2. Bryson, S., Levy, D.: Central schemes for multi-dimensional Hamilton-Jacobi equations. SIAM J. Sci. Comput. (to appear).

3. Bryson, S., Levy, D. (2003): High-Order Central WENO Schemes for Multi-dimensional Hamilton-Jacobi Equations. SIAM J. Numer. Anal., 41, 1339-1369.

4. Bryson, S., Levy, D. (2003): High-order semi-discrete central-upwind schemes for multidimensional Hamilton-Jacobi equations. J. Comp. Phys., 189, 63-87.

5. Crandall, M.G., Ishii, H., Lions, P.-L. (1992): User's guide to viscosity solutions of second order partial differential equations. Bull. Amer. Math. Soc., 27, 1-67.

6. Crandall, M.G., Lions, P.-L. (1983): Viscosity solutions of Hamilton-Jacobi equations. Trans. Amer. Math. Soc., 277, 1-42.

7. Jiang, G.-S., Peng, D. (2000): Weighted ENO schemes for Hamilton-Jacobi equations. SIAM J. Sci. Comp., 21, 2126-2143.

8. Jiang G.-S., Shu C.-W. (1996): Efficient implementation of weighted ENO schemes. J. Comp. Phys., 126, 202-228.

9. Kossioris G., Makridakis Ch., Souganidis P.E. (1999): Finite volume schemes for HamiltonJacobi equations. Numer. Math., 83, 427-442.

10. Kurganov, A., Noelle S., Petrova G. (2001): Semi-discrete central-upwind schemes for hyperbolic conservation laws and Hamilton-Jacobi equations. SIAM J. Sci. Comp., 23, 707-740.

11. Kurganov, A., Tadmor, E. (2000): New high-resolution semi-discrete central schemes for Hamilton-Jacobi equations. J. Comp. Phys., 160, 720-742.

12. Lin, C.-T., Tadmor, E. (2000): $L^{1}$-stability and error estimates for approximate HamiltonJacobi solutions. Numer. Math., 87, 701-735.

13. Lin, C.-T., Tadmor, E. (2000): High-resolution non-oscillatory central schemes for approximate Hamilton-Jacobi equations. SIAM J. Sci. Comp., 21, 2163-2186.

14. Lions, P.L. (1982): Generalized solutions of Hamilton-Jacobi equations. Pitman, London

15. Lions, P.L., Souganidis, P.E. (1995): Convergence of MUSCL and filtered schemes for scalar conservation laws and Hamilton-Jacobi equations. Numer. Math., 69, 441-470. 
16. Liu, X.-D., Osher, S., Chan, T. (1994): Weighted essentially non-oscillatory schemes. J. Comp. Phys., 115, 200-212.

17. Osher, S., Sethian, J. (1988): Fronts propagating with curvature dependent speed: algorithms based on Hamilton-Jacobi formulations. J. Comp. Phys., 79, 12-49.

18. Osher, S., Shu, C.-W. (1991): High-order essentially nonoscillatory schemes for HamiltonJacobi equations. SIAM J. Numer. Anal., 28, 907-922.

19. Souganidis, P.E. (1985): Approximation schemes for viscosity solutions of Hamilton-Jacobi equations. J. Diff. Equations, 59, 1-43.

20. Zhang, Y.-T., Shu, C.-W. (2002): High-order WENO schemes for Hamilton-Jacobi equations on triangular meshes. Siam J. Sci. Comput., 24, 1005-1030. 